\title{
Task-Related Sensorimotor Adjustments Increase the Sensory Range in Electrolocation
}

\author{
Federico Pedraja, ${ }^{1}$ Volker Hofmann, ${ }^{1,2}$ Julie Goulet, ${ }^{1}$ and ${ }^{\oplus}$ Jacob Engelmann ${ }^{1}$ \\ ${ }^{1}$ AG Active Sensing, Faculty of Biology/Cluster of Excellence Cognitive Interaction Technology, Bielefeld University, D-33501 Bielefeld, Germany, and \\ ${ }^{2}$ Department of Physiology, McGill University, Montreal, Quebec H3G 1Y6, Canada
}

Perception and motor control traditionally are studied separately. However, motor activity can serve as a scaffold to shape the sensory flow. This tight link between motor actions and sensing is particularly evident in active sensory systems. Here, we investigate how the weakly electric mormyrid fish Gnathonemus petersii of undetermined sex structure their sensing and motor behavior while learning a perceptual task. We find systematic adjustments of the motor behavior that correlate with an increased performance. Using a model to compute the electrosensory input, we show that these behavioral adjustments improve the sensory input. As we find low neuronal detection thresholds at the level of medullary electrosensory neurons, it seems that the behavior-driven improvements of the sensory input are highly suitable to overcome the sensory limitations, thereby increasing the sensory range. Our results show that motor control is an active component of sensory learning, demonstrating that a detailed understanding of contribution of motor actions to sensing is needed to understand even seemingly simple behaviors.

Key words: active sensing; behavior; closed-loop control; electrolocation; electroreceptors; learning

\section{Significance Statement}

Motor-guided sensation and perception are intertwined, with motor behavior serving as a scaffold to shape the sensory input. We characterized how the weakly electric mormyrid fish Gnathonemus petersii, as it learns a perceptual task, restructures its sensorimotor behavior. We find that systematic adjustments of the motor behavior correlate with increased performance and a shift of the sensory attention of the animal. Analyzing the afferent electrosensory input shows that a significant gain in information results from these sensorimotor adjustments. Our results show that motor control can be an active component of sensory learning. Researching the sensory corollaries of motor control thus can be crucial to understand sensory sensation and perception under naturalistic conditions.

\section{Introduction}

Exploratory behavior is a crucial substrate for learning (Loewenstein, 1994). As movements of the animals influence the sensory input, reorganizing the motor patterns with respect to recent experience may contribute to learning, thereby improving behavior. Analyzing these modifications can thus reveal how motor action contributes to learning (Wolpert and Landy, 2012; O'Hora et al., 2013) and aid decision-making through action selection (Charlesworth et al., 2011; O’Hora et al., 2013; Zgonnikov et al., 2017).

Received May 14, 2019; revised Nov. 9, 2019; accepted Nov. 18, 2019.

Author contributions: F.P., V.H., and J.E. designed research; F.P. performed research; J.G. contributed unpublished reagents/analytic tools; F.P., J.G., and J.E. analyzed data; F.P., V.H., and J.E. wrote the paper.

This work was supported by the Cluster of Excellence Cognitive Interaction Technology "CITEC" (Grant EXC 277) and the Deutsche Forschungsgemeinschaft (Grant EN 826/5-1).

The authors declare no competing financial interests.

Correspondence should be addressed to Jacob Engelmann at Jacob.Engelmann@uni-bielefeld.de.

https://doi.org/10.1523/JNEUROSCI.1024-19.2019

Copyright $\odot 2020$ the authors
While the variability of motor behavior may facilitate motor learning by widening the search space from which behaviors are instantiated (Brainard and Doupe, 2013; Wu et al., 2014), the same variability can set bounds on the task optimization of motor control (van Beers et al., 2002). This is particularly evident in active sensory systems, where the sensory input directly depends on the motor output. Here the strong sensorimotor dependencies may be exploited by an animal to adjust motor behavior to improve not only the motor, but also the sensing efficiency (Friston, 2010; Little and Sommer, 2013; Gordon et al., 2014).

We here investigated how sensorimotor behavior changes while Gnathonemus petersii, a pulse-type weakly electric fish, learned a detection task. During active electrolocation, these fish obtain sensory information through brief discharges of a specialized electric organ in their tail [electric organ discharge (EOD)]. The discharge rate is under top-down control and changes in a context-dependent manner (Post and von der Emde, 1999; Caputi et al., 2003). Each emitted EOD creates a 3D electric field around the fish which is perturbed by nearby objects (Lissmann 
and Machin, 1958). Also the motion of the animal can perturb the electric field (e.g., tail movement; Sawtell et al., 2006), both of which are perceived by electroreceptors in the skin of the fish. To discriminate between the predictable (reafferent) and unpredictable (ex-afferent) components of the sensory input, weakly electric fish are known to rely on a sophisticated neuronal circuitry (Sawtell et al., 2005; Bell et al., 2008), which enables them to analyze their nearby environment.

Not all (reafferent) sensory consequences of behavior must be unfavorable, however: similar to other organisms (Poteser and Kral, 1995; Kern et al., 2001), weakly electric fish exhibit a variety of stereotyped behaviors (Toerring and Belbenoit, 1979; Toerring and Moller, 1984; Nelson and Maciver, 1999; Hofmann et al., 2014). Recent studies have revealed that behaviorally relevant sensory information can emerge from such strongly patterned sensorimotor behaviors (i.e., weakly electric fish actively exploit these sensorimotor dependencies; Hofmann et al., 2017; Pedraja et al., 2018).

The ability to control the timing of sensory sampling while at the same time being able to shape the properties of the sensory input through their motor behavior makes weakly electric fish particularly suitable to study how changes in exploratory behaviors can guide sensory-driven learning efficiently.

We here focused on a reinforced object detection task and found that performance was progressively enhanced by consistent changes of the motor patterns. These changes resulted in an increased sensory range. Our results add further support to the idea that weakly electric fish actively improve sensing capabilities by selecting purposeful components from their motor repertoire and focus their electric attention in a goal-directed manner. Such behavioral control of the sensory input might contribute to improving neuronal stimulus detection and encoding, as we found neuronal performance to be relatively poor at the level of the medulla.

\section{Materials and Methods}

\section{Animals}

Wild-caught $G$. petersii of either sex were obtained from a commercial fish dealer (Aquarium Glaser) and housed in communal $400 \mathrm{~L}$ aquaria. The water temperature in these aquaria and the setup was $25 \pm 1{ }^{\circ} \mathrm{C}$ at a conductivity of $100 \pm 5 \mu \mathrm{S} \cdot \mathrm{cm}^{-1}$ and a $12 \mathrm{~h} \mathrm{light/dark} \mathrm{cycle.} \mathrm{Fish} \mathrm{were}$ fed with bloodworms. All procedures for animal maintenance and preparations comply with the current animal protection law of the Federal Republic of Germany and have been approved by the local authorities (Landesamt für Natur, Umwelt und Verbraucherschutz NordrheinWestfalen: 87-51-04.2010.A202).

\section{Behavior}

Training setup. Five G. petersii (length, $11 \pm 1 \mathrm{~cm}$ ) housed in separate experimental tanks and fed with bloodworms to satiation three times per week before the beginning of the behavioral experiment. The experimental tanks $(120 \times 50 \times 50 \mathrm{~cm})$ were divided in a living area $(60 \times 50 \mathrm{~cm}$; water level, $30 \mathrm{~cm})$ that was separated by a plastic gate from the experimental area $(60 \times 50 \mathrm{~cm}$; water level, $8-10 \mathrm{~cm})$. The floor in the experimental area was $20 \mathrm{~cm}$ above the floor of the living area, which confined the movements of the animal in the experimental area into two dimensions. A plastic plate divided the proximal end $(20 \mathrm{~cm})$ of the experimental arena into two compartments. Perpendicular to this plate, a 1-cm-wide plastic strip marked the entry to the compartments on the floor. A metal cube $(2 \times$ $2 \times 2 \mathrm{~cm}$ ) was placed on the floor at the decision line, centered in front of the cued compartment where it served as a cue to the rewarded compartment $(S+)$. Experiments were performed in darkness $(<0.1$ lux measured above the water level) and videotaped from the top (60 frames/s; Marlin F-131 and F-033, AltaVision) using infrared illumination (880 $\mathrm{nm}$ ) from below. This wavelength is beyond the perceptual range of this species (Ciali et al., 1997). EODs were recorded differentially (custom- built electrode array, $0.6-40 \mathrm{kHz}$ bandpass) and stored as events (PC audio card, 12 bit, $10 \mathrm{kHz}$ ) alongside acquired videos.

Experimental design, video tracking, and statistical analysis. Animals first learned to swim through the opened gate to the proximal end of the arena to receive food. Once fish did this reliably, training commenced, and videos of each trial were acquired. Each trial started by opening the gate and ended with the fish swimming back to the living area. Crossing of the decision line was scored as a choice, and correct choices were rewarded with food. After the fish returned to the living area, the gate was closed. Which of the two compartments was cued was determined in a pseudorandom fashion (Gellermann, 1933). The cube was removed from the tank and repositioned after each trial, even when the same compartment was cued in consecutive sessions. Training was performed $6 \mathrm{~d} /$ week with one session of 20-30 trials per day. When the performance reached $80 \%$ correct trials in six consecutive sessions, we considered learning to be completed. We then separated the data of each fish into three learning stages: stage I contained the first six sessions where performance was $<60 \%$ (511 trials from five animals); stage II contained the consecutive six sessions (530 trials) where performance was $>60 \%$ and $<80 \%$; and stage III comprises the first six session after the fish exceeded $80 \%$ performance (592 trials).

In addition, we determined the sensory detection range after learning was completed by gradually increasing the distances of the cue from the decision line $(2-12 \mathrm{~cm})$. At each distance, $20-40$ test trials were conducted before increasing the test distances. Similar to the training procedure, sessions were performed $6 \mathrm{~d}$ /week with 20 control and 10 test trials per session. The detection limit was determined as the object distance where the sigmoidal fit to the performance reached $75 \%$.

Using a background subtraction approach the center of mass of the animals was determined offline using custom-written MATLAB routines (64 bit; R2016b, MathWorks). The posture of the animal was obtained by applying a third-order polynomial fit through the midline of the body. Head and tail positions were determined based on the spindle-like shape of the body of the fish with the head being closer to the center of mass of the body. The position of the object was also tracked. From the change of the position of the animal between consecutive frames, we determined the $2 \mathrm{D}$ kinematics (i.e., thrust, slip, and yaw velocity), which were used for the behavioral classification (for more information, see Hofmann et al. (2014)). In trials where the cue was in the left compartment, the data were mirrored along the long axis of the arena to have movement prototypes in consistent relation to the cued compartment.

To quantify the spatial distribution of behaviors the arena was binned (bin size $1 \times 1 \mathrm{~cm}$ ). The distance to the cube was measured as the Euclidean distance between the head of the fish and the object. In trials where the fish chose the wrong compartment, distance was calculated with respect to the virtual object position (i.e., we assume the object to be present in the compartment the fish had erroneously chosen). To illustrate the average trajectories per fish and learning stage, we obtained the mean direction in which fish passed from one spatial bin to the next and the corresponding vector strength $z\left(z=r^{2} \cdot N\right)$, with $N$ being the number of elements in the bin and $r^{2}$ the Rayleigh coefficient of angular dispersion). Based on these values, the average gradient of the trajectories was visualized using the streamline function in MATLAB. This only served as a visualization tool, while all analysis is based on single trajectories. Sampling density was calculated as the number of EODs emitted per distance traveled (EOD count $\cdot \mathrm{cm}^{-1}$ ) using swim speed and the EOD rate per frame. To calculate attracting sets, spatial maps were generated from all trajectories. From each trajectory, the first coordinate received a weight of +1 and the last coordinate a weight of -1 . Weights for coordinates in between were linearly interpolated based on traveled time and distance. For each session, we superimposed all weighted trajectories, resulting in cumulative $2 \mathrm{D}$ maps. The size of the attracting sets was defined as the area in which data fell below a threshold that was defined by 2 standard deviations (the SD was calculated based on all values for all fish) above the minimum value of the map.

Transient increases of the EOD rate (E-scans) were detected from the $z$-scored first derivative of the EOD frequencies. The variance and mean for the $z$-transform were based on pooled data of a given training trial. 
A

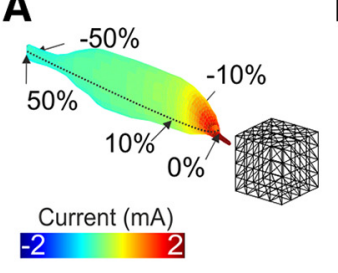

B

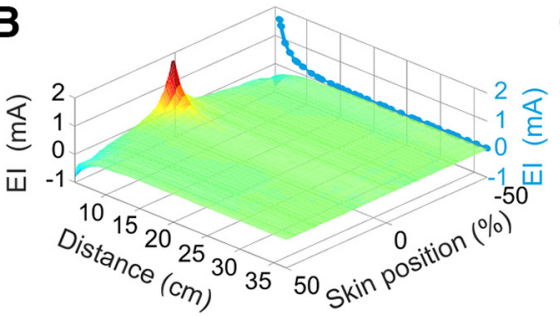

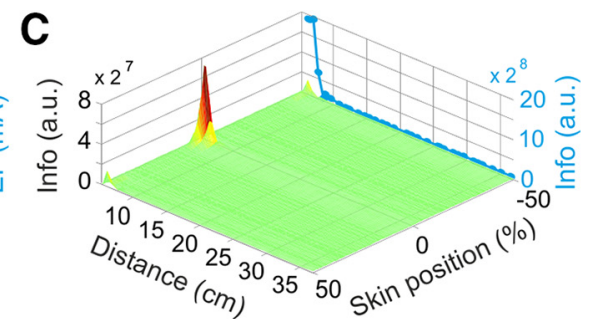

Figure 1. Electric image analysis. $A$, View of the geometric model of the fish body with the color depicting the transcutaneous current expressed as the difference between the perturbed and unperturbed electric field. The stippled black line spanning the body of the fish indicates the positions where the Els were analyzed. $\boldsymbol{B}$, Spatial sequence of the electric images calculated along the midline of the fish for a complete approach sequence. The position of the El is expressed relative to the length of the fish with the snout being the zero position (see $A$ ). The blue line represents the El peak values as a function of distance to the object. $\boldsymbol{C}$, As in $\boldsymbol{B}$ but for the Fisher information. The blue line shows the Fisher information integrated over each image as a function of distance.

Accelerations exceeding a $z$-value of 1.5 were defined as E-scans, and their location was defined by the position at which the E-scan began.

All statistical analyses were performed using MATLAB and PAST 3 (Paleontological Statistics Software Package for Education and Data Analysis, version 3.1). The normality of data was examined by the Shapiro-Wilk test and tested for homogeneity of variance with the Levene's test (significance criterion, $p \leq 0.05$ in both cases). The appropriate parametric test ( $t$ test for linear regressions) or nonparametric test (Wilcoxon-signed rank test, Mann-Whitney pairwise test, or KruskalWallis test) was used accordingly and is indicated in the Results and in captions throughout. Data used for multiple comparisons was corrected post hoc (Bonferroni's correction) when necessary using $p \leq 0.05$ for significance. Transition probabilities of super-prototypical movements (SPMs) were tested using Pearson's $\chi^{2}$ test.

Behavioral classification. We classified the behavior based on clustering algorithms, as previously described (Braun et al., 2010; Geurten et al., 2010; Hofmann et al., 2014). Kinematics were clustered hierarchically (Ward's criterion), and the quality and stability of the clusters were assessed to determine the optimal number of clusters. Next, data was clustered applying a k-means algorithm with the optimal number of clusters regarding quality and stability ( 10 in our case). The resulting centroid of each cluster (thrust, slip, and yaw velocities) was used to express the kinematic properties of identified clusters that we here refer to as "prototypical movements" (PMs). PMs resemble the basic motor components of the recorded behavior on a frame-by-frame basis. PMs with yaw velocities above half of the maximum observed yaw velocity $\left(64^{\circ} \cdot \mathrm{s}^{-1}\right)$ were defined as left- or right-turn PMs, respectively. PMs where the thrust velocities exceeded $75 \%$ of the maximum observed thrust $(43.5$ $\mathrm{cm} \cdot \mathrm{s}^{-1}$ ) were considered high-thrust PMs, while PMs with thrust values $<25 \%$ of this maximum were considered low-thrust PMs. Using this categorization, we calculated thrust- and turn-triggered averages of electromotor behavior (E-scans) and the quality of the sensory input [Fisher information (FI); see below]. From these averages, we compared the E-scan probability and Fisher information for the $100 \mathrm{~ms}$ surrounding different PMs.

To characterize behavior on larger timescales, we calculated the transition probabilities between PMs. The probabilities were processed in a hidden Markov model to determine the most frequent sequences of four consecutive PMs, termed "super-prototypical movements" (Braun et al., 2010). Their complexity was reduced by combining SPMs with comparable PM composition (e.g., merging PM sequence 1-1-1-2-1-1-2-2 with PM sequence 2-2-1-2-2-2-1-1). The 300 most frequent SPMs were then further analyzed with respect to where (arena) and when (learning stage) they occurred. We then focused on the SPMs that changed the most between learning stages I and III. That is, in each area of the arena we obtained the SPMs with the strongest drop in their recruitment probability between stages I and III as well as those that showed the strongest increase in their recruitment probability when comparing learning stage III against stage I. SPMs were again categorized by their yaw and thrust velocities. In turn-dominated SPMs, the mean yaw exceeded at least half of the maximum mean yaw velocity of all SPMs $\left(29^{\circ} \cdot \mathrm{s}^{-1}\right)$. The remaining SPMs were grouped based on the maximum average thrust velocity $\left(42 \mathrm{~cm} \cdot \mathrm{s}^{-1}\right)$, resulting in low-thrust $(<25 \%$ of maximum), medium- thrust (between $25 \%$ and $75 \%$ of maximum), and high-thrust ( $>75 \%$ of maximum) SPMs. The spatial distribution of different SPMs was analyzed based on the $x$ and $y$ coordinates of the first PM in each SPM sequence. This was accumulated and fitted with a 2D Gaussian. For visualization, we show the area of these fits where the SPM probability is $>0.1 \%$. On average, $89 \%$ of the individual data falls within this contour (range, 77-96\%).

Electric image model. Electric images (EIs) were computed with software developed by Rother Malamud (2003). This approach was verified and used in previous studies (Rother et al., 2003; Migliaro et al., 2005; Sanguinetti-Scheck et al., 2011; Hofmann et al., 2013, 2017; Pedraja et al., 2014). The model consists of a geometric reconstruction of the body of the fish and a calculation of the transcutaneous field by solving the Poisson equation for the boundary of the fish using the boundary element method. Briefly, this method determines the boundary electrical distributions solving a linear system of $\mathrm{M} \cdot \mathrm{N}$ equations for $\mathrm{M}$ poles and $\mathrm{N}$ nodes, with the unknown variables being the transepithelial current density and potential at each node (Pedraja et al., 2014). The transepithelial current density and potential is calculated for each node and linearly interpolated for the triangles defined by the nodes, forming the geometry of fish and objects (Fig. 1A). From this, the electric images for each trajectory were calculated as the difference between the amplitude of the positive EOD peak in the presence and absence of the object (Fig. 1B).

Fisher information analysis. To estimate how informative the electric images (sensory input) are with respect to an estimate of the location of the object, we calculated the Fisher information. As detailed previously (Silverman et al., 2013; Miller et al., 2016), the formal definition of Fisher information when estimating an unknown parameter $\theta$ is as follows:

$$
I(\theta)=\int_{v}\left(\frac{\partial \mathrm{p}(v \mid \theta)}{\partial \theta}\right)^{2} \frac{1}{p(v \mid \theta)} d v,
$$

where $p(v \mid \theta)$ is the probability of observing $v$ given $\theta$. The Fisher information then is calculated as the integral over all possible observable $v$. In our case, the measurement $v$ depends on a function $Y(\cdot)$ of the parameter $\theta$, as follows:

$$
v=\mathrm{Y}(\theta, x)+\delta
$$

where $Y(\theta, x)$ is the electric current density at the nodes of the fish at the location of the fish $(x)$, and $\delta$ is zero mean noise with variance $\sigma^{2}$. The Fisher information then is calculated as follows:

$$
I(\theta, x)=\left(\frac{\partial \mathrm{Y}(\theta, x)}{\partial \theta} \cdot \frac{1}{\sigma}\right)^{2} .
$$

The best estimate $(\theta)$ of the position of the cube is obtained when the partial derivative $\left(\frac{\partial \mathrm{Y}(\theta, x)}{\partial \theta}\right)$ is maximal. When approaching the cube, this will occur at the position where the slope in the electric field is highest. $I(\theta, x)$ can be considered as the change of the distributed information that an electric image provides on the position of the cube with respect to the variance of the noise. Here the noise is based on the first EI 
of each trajectory. Integrating the distributed information $I(\theta, x)$ over the nodes of the modeled fish yielded in a single measure of information at each location (Fig. 1C, blue line).

Artificial trajectories. To evaluate the dependence of the Fisher information on the trajectories to the cube, artificial trajectories to the target were simulated. In these, the fish body was kept straight, and the swim speed and EOD rate were constant. We chose two trajectories where the fish targeted the object lateral to its center of mass, and one where it approached frontally. For both, we then calculated the electric images and Fisher information. By concatenating electric images from the frontal and lateral approach, we simulated an undulatory trajectory in which the electric image moves over the head region of the fish. A qualitative comparison of the Fisher information reached by these different approaches to the target was then conducted.

\section{Physiology}

Surgery. Electrophysiological experiments were performed in 16 G. petersii (length, $11 \pm 2 \mathrm{~cm}$ ). Before the experiments, fish were anesthetized in buffered 3-aminobenzoic acid ethyl ester methanesulfonate salt $\left(0.1 \mathrm{~g} \cdot \mathrm{L}^{-1}\right.$; MS-222, Sigma-Aldrich), immobilized with an intramuscular injection of $20 \mu \mathrm{l}$ of pancuronium bromide (1:100 in Ringer's solution; Braun-Melsungen) and transferred to a holder in the experimental tank $(60 \times 40 \times 15 \mathrm{~cm})$. During surgical procedures, fish were respirated with MS-222 solution for anesthesia $\left(0.05 \mathrm{~g} \cdot \mathrm{L}^{-1}\right)$. In addition, the surgery site was treated with a local anesthetic (Xylocaine 2\%; Astra Zeneca). Afterward, the skin at the dorsal part of the cranium was removed and the head was fixed to a plastic rod (Formatray, Kerr). Then the craniotomy was carried out above the caudal end of the cerebellum.

After surgery, fish were respirated with freshwater, and the tank water was exchanged to remove any MS-222 residuals. Spinal cord activity (see below) resumed typically within 10-15 min following the end of anesthesia. In all cases, water conductivity was $100 \pm 5 \mu \mathrm{S}$ and the respiration rate was $40 \mathrm{ml} \cdot \mathrm{min}^{-1}$.

EOD playback. Paralysis blocks the myogenic electric organ, but the descending command signal of the pacemaker nucleus persists and can be used to trigger a synthetic EOD at the time and amplitude of the natural EOD. Amplitude and timing of the EOD of each individual was measured before the surgery by sedating the fish with Etomidate-Lipuro ( $400 \mu \mathrm{g} \cdot \mathrm{L}^{-1}$; Braun Melsungen) and recording the command signal and the EOD. The timing of the command signal was measured with a hookshaped electrode around the electric organ (amplification, $\times 500$; MA 103, Electronic Workshop, University Cologne; bandpass filtering, $1 \mathrm{~Hz}$ to $1 \mathrm{kHz}$ ). The natural EOD was measured (amplification, $\times 50$; MA 103 , Electronic Workshop, University Cologne; bandpass filtering, $10 \mathrm{~Hz}$ to $30 \mathrm{kHz}$ ) with a dipole electrode placed in the water close to the left eye and oriented perpendicular to the main body axis of the fish. As a playback, a prerecorded EOD (DG1000 waveform generator, RIGOL Technologies; A385 Stimulus Isolator, WPI) was issued via two silver wires, one of which was implanted into the EO and the other was placed caudal to the tail of the fish. The playback was triggered by the command signal with the predetermined delay and set to match the amplitude of the natural EOD.

Electrophysiological recording. Tungsten electrodes $(3 \pm 2.5 \mathrm{M} \Omega$; 7-Channel Single Electrode System System Eckhorn Microdrive and SUA Amplifier, and $1000 \times$ MTREC, Thomas Recording) were inserted through the posterior part of the cerebellum toward the medial zone of the electrosensory lateral line lobe (ELL). Electrode depth and the local field potential $(10 \mathrm{~Hz})$ were monitored until the plexiform layer of the ELL was reached. Then filter settings were adjusted (bandpass, $100-3000$ $\mathrm{Hz}$ ), and electrodes were carefully advanced to isolate single-unit activity. Recordings were digitized ( $25 \mathrm{kHz}$ sampling rate, 12 bit resolution; Spike 2 version 6 and Micro 1401-MKII, CED) and stored for offline analysis.

Stimulation. We recorded from principal cells of the medial zone of the ELL. These cells show either an excitatory response to an increase in local EOD amplitude (E-cells; $n=8$ ) or an inhibitory response (I-cells; $n=$ 12). Receptive fields are heterogeneous in size and location on the body (Metzen et al., 2008), and the center was determined with a small dipole electrode over which a local playback of the EOD was issued while moving it alongside the animal. Receptive fields were predominantly located on the head and first third of the trunk of the animals. Afterward, the global EOD mimic was again presented for at least 1 min before presenting a cube $(2 \times 2 \times 2 \mathrm{~cm}$; metal for E-cells, plastic for I-cells $)$ in the center of the respective receptive field. The object was presented for $1 \mathrm{~min}$ during which typically $30-70$ EODs were registered (median EOD inter$\mathrm{val}, 357 \mathrm{~ms} ; 25 \%$ percentile, $224 \mathrm{~ms}$ ). The distance of the object surface relative to the skin surface was randomly varied between 1 and $35 \mathrm{~mm}$ using a micromanipulator, and in between each stimulus presentation we recorded $1 \mathrm{~min}$ of ongoing activity without the object being present.

Analysis. Spikes were extracted and sorted using a wavelet separation with following principal component analysis and cluster analysis (Spike 2, CED). Further analysis included only well isolated single units and was performed with custom-written routines in MATLAB. ELL units fire a burst of spikes following each EOD with changes in the burst parameters encoding for electrosensory stimuli. We characterized the mean firing rate, maximum firing rate, and latency of each burst. The mean firing rate was determined as the number of spikes following the EOD within a 150 $\mathrm{ms}$ window. For this, only spikes within a given EOD interval were included in the analysis. Results were qualitatively similar for shorter spike count windows (i.e., 100 or $50 \mathrm{~ms}$ ). The maximum firing rate was determined as the peak of the convolved firing rate (15 ms boxcar convolution). Burst latency was determined as the latency of the first elicited spike relative to the EOD time.

We used a receiver operating characteristic (ROC) analysis to quantify if and at which distance neuronal activity would enable the detection of the object. For this, we used the probability distributions of the burst parameters as determined for stimulated and ongoing activity to calculate the probability of true-positive and false-positive hits. The area under the curve (AUC) of the ROC was used as a sensitivity measure at a given distance from the cube. This measure was plotted as a function of distance and fitted by a sigmoidal function. The detection limit was determined as the point where the fit fell below a sensitivity of 0.7 . The population average was assessed through calculating a sliding bin (bin width, $2 \mathrm{~cm}$ ) of the raw sensitivity data.

\section{Results}

In the current study, we investigated the object detection performance of the weakly electric fish $G$. petersii. First, we will present results from single-unit recordings of medullary electrosensory principal neurons. Based on these recordings, we establish the distances at which physical objects can be detected neuronally. We then contrast the neuronal results with the behavioral performance levels, emphasizing the often underestimated contribution of sensorimotor interactions for perception.

\section{Detection limits based on neuronal recordings in ELL}

The ELL is the first central nucleus of the ascending electrosensory pathway and receives converging input from electroreceptor afferents (Bell et al., 1989, 2005; Hollmann et al., 2016). Afferent responses are directly related to the local amplitude modulation of the EOD; however, even large conductive objects modulate afferent activity only within a range of $\sim 10 \mathrm{~mm}$ (Szabo and Hagiwara, 1967; Gómez et al., 2004). It is known that ELL principal cells can have large and complex receptive fields (Metzen et al., 2018); their detection threshold for physical object distance, however, has not been established.

We recorded the responses of 20 isolated ELL principal cells in immobilized animals $(n=16)$ to objects (metal and plastic cubes; edge length, $2 \mathrm{~cm}$ ) presented within the receptive fields center at varying distances up to $40 \mathrm{~mm}$ from the skin of the fish (Fig. $2 \mathrm{~A}$ ). ELL principal cells typically issued a short burst of spikes following each EOD (Fig. 2B). We characterized these bursts in terms of their latency, their maximum firing rate, and the mean firing rate (see also Materials and Methods), which are the parameters that typically change in response to a presented stimulus. To evaluate object detection, we analyzed responses using an ROC. For this, 
A

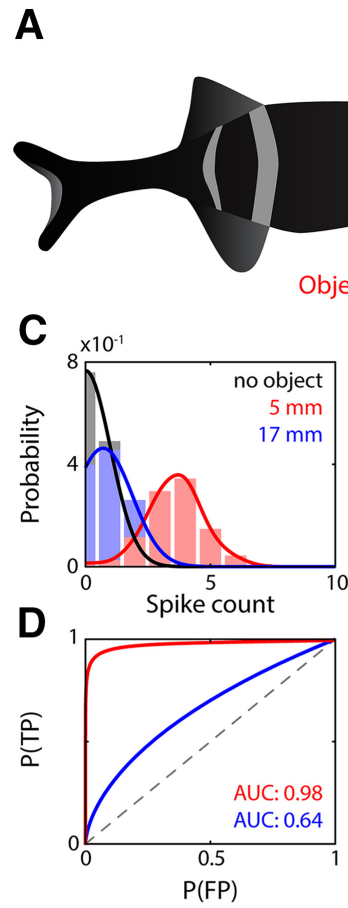

$\mathbf{F}$

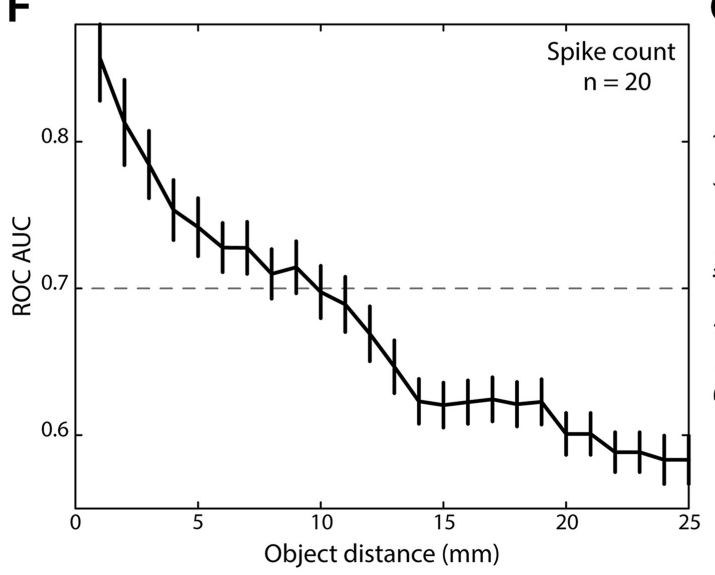

E
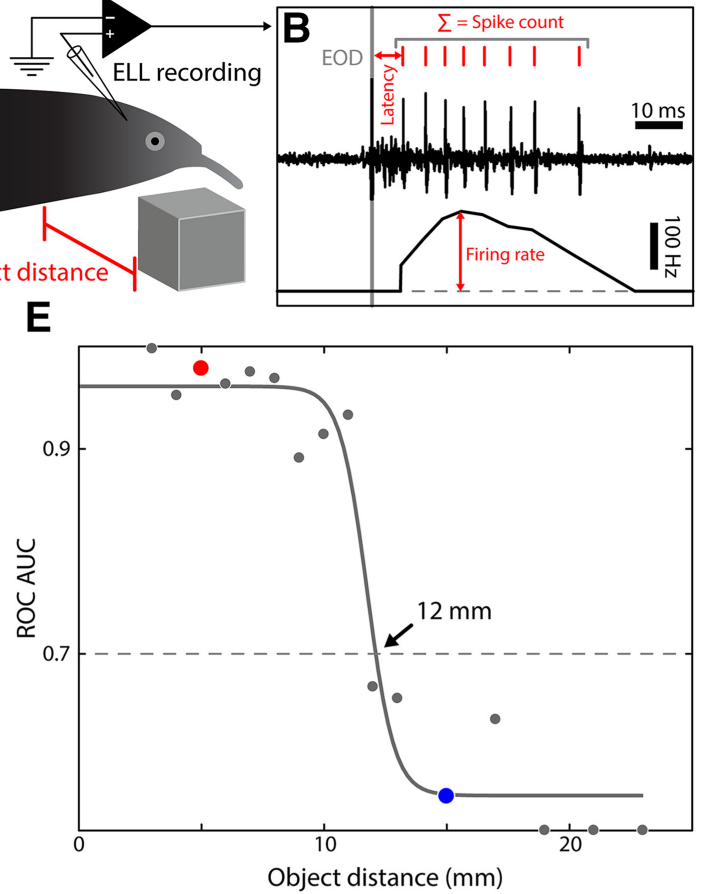

$\mathbf{G}_{30}$

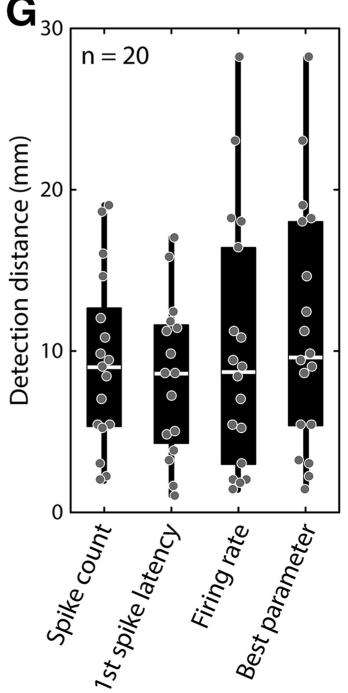

Figure 2. Object detection capabilities of hindbrain neurons. $A$, The activity of ELL principal neurons $(n=20)$ was recorded in immobilized G. petersii. Ongoing activity was recorded before each stimulus presentation (metal or plastic cube; side length, $2 \mathrm{~cm}$ ). $\boldsymbol{B}$, Neuronal activity (middle, black) typically consisted of a burst of action potentials following the EOD (gray vertical line). For each burst, we measured the number of spikes within $150 \mathrm{~ms}$ after each EOD (top red), the latency of the first spike (red arrow), and the peak of the convolved firing rate (bottom, black trace and red arrow). Only spikes within one EOD cycle were included (median EOD rate, $357 \mathrm{~ms}$ ). C, Probability distributions (solid lines) for the measured parameters (here spike count) of the responses of an example E-unit were stimulated with a metal object at $17 \mathrm{~mm}$ (blue) and $5 \mathrm{~mm}$ (red) as well as ongoing activity (black). D, ROCs (see Materials and Methods) obtained for the data shown in C. For each stimulation distance, the probability of a true-positive $P(\mathrm{TP})$ classification was calculated as a function of the probability of a false-positive $P(F P)$ classification. The AUC was assessed. E, ROC sensitivity (ROC AUC) as a function of distance of the object for an exemplary E-unit. The detection distance of all units was determined as the point where a sigmoidal fit to the data exceeded an AUC of 0.7 . In this example, the detection distance was 12 $\mathrm{mm}$. Red and blue dots correspond to the data shown in $\boldsymbol{C}$ and $\boldsymbol{D}$. $\boldsymbol{F}$, Average ROC data for 20 single units based on spike count probability. $\mathbf{G}$, Distribution of detection distances of all units. While some neurons had detection distances up to $28 \mathrm{~mm}$, the average detection threshold was $<10 \mathrm{~mm}$ for all parameters. Gray dots show the detection distances of individual neurons.

response distributions were obtained over several instances of EOD emission and compared with ongoing activity (Fig. $2 C$, top, object at $5 \mathrm{~mm}$ (red) vs ongoing activity (black); bottom, object at $17 \mathrm{~mm}$ (blue) vs ongoing activity (black)). ROC sensitivity, quantified by the AUC of the ROC (Fig. 2D), decreased in a sigmoidal fashion (Fig. 2E, solid line).
Detection distance was defined as the point where the sigmoidal fit fell to $\leq 0.7$ (Fig. 2E, arrow). On average, this distance was within the range of $10 \mathrm{~mm}$ (Fig. $2 F$; population average, $n=20$ neurons analyzed with spike counts), regardless of the parameter used to analyze responses (Fig. $2 G$; mean $\pm \mathrm{SD}$ : spike count, $9.3 \pm 5.4$ $\mathrm{mm}$; latency, $8.3 \pm 4.8 \mathrm{~mm}$; firing rate, $10.0 \pm 7.9 \mathrm{~mm})$. Threshold in these groups (different parameters analyzed) were not significantly different (KruskalWallis test, $p=0.90$ ). In addition, the detection distance did not increase significantly when the best parameters per cell were pooled (Kruskal-Wallis test, $p=$ 0.76). While the responses of some individual neurons allowed for object detection up to a distance of $28 \mathrm{~mm}$, the average detection threshold across the recorded population was poor and within a range of $\sim 10 \mathrm{~mm}$.

\section{Task acquisition is paralleled by sensorimotor alterations}

We trained five fish to detect a metal cube of the same size as used in physiology (Fig. 3A; and Materials and Methods). All fish reached a stable performance within $22.8 \pm 3.8$ sessions (Fig. $3 B$ and Fig. 3-1, available at https://doi.org/ 10.1523/JNEUROSCI.1024-19.2019.f3$1)$. Based on the performance, the data were split for further analyses (Fig. 3B; stage I, <60\% (light cyan); stage II, 60$80 \%$ (dark cyan); stage III, $>80 \%$ (violet)). Figure $3 C$ depicts the average movements (black lines) of one fish during the three different stages. The mean heading direction within each spatial bin $\left(1 \mathrm{~cm}^{2}\right)$ is shown as a color code (left to right as blue to red), showing that trajectories in phase I (Fig. 3C) were mainly straight, corresponding to the chancelevel performance in this stage (Fig. 3B, light cyan). With learning, trajectories showed a characteristic increase of right turns (red) in area II (Fig. 3D,E). While traversing the arena, fish gradually decreased their speed (Fig. $3 F$ ) with swim speeds being slightly higher (Fig. $3 F$ ). In addition, the dispersion of the trajectories (distribution along the shorter axis of the arena) decreased with learning, showing that fish preferentially swam along the middle of the arena in later learning stages (Fig. $3 G)$. Altogether, these changes resulted in a better alignment of the heading of the fish with the object (Fig. $3 \mathrm{H}-$ J; mean direction: $321^{\circ}, 330^{\circ}$, and $333^{\circ}$ for stages I-III; William-Watson test for difference in orientation, $p<$ 0.001 ; mean vector strength, $0.72,0.79$, and 0.86 for stages I-III, respectively). Note that fish were rewarded at the end of the arena (i.e., only after having passed the object). As such, they were not forced to target the object. 
These results, centered on the general motor behavior, already show that improved performance went along with adjustments of the motor behavior.

\section{Motor behavior is altered by a differential recruitment of basic kinematic components}

The 10 prototypical movements revealed here (Geurten et al., 2010; Hofmann et al., 2014) were either thrust-dominated PMs (PMs 1-4) or turn-dominated PMs (right-turn PMs, 6, 8, and 10; left-turn PMs, 5, 7, and 9; Fig. 4A). The separation in $10 \mathrm{PMs}$ did not change with learning. While the frequency of PM 1 dropped by $29 \%$ when comparing stages I and III, the frequency of the other PMs did not change as strongly with learning (average variation between stages I and III, $7.5 \pm$ $3.8 \%)$. What changed with learning, however, was the kinematic composition of the behavioral sequences (i.e., SPMs; Fig. $4 B$ ). We here focus on the nine SPMs with the strongest change of occurrence between the learning stages III and I in correct trials (see Materials and Methods). These were also among the most frequently occurring SPMs in absolute terms (for details, see Fig. 4-1, available at https://doi.org/10.1523/JNEUROSCI. 1024-19.2019.f4-1). Learning led to a reduction of thrust-dominated SPMs (Fig. $4 C$, shades of black) and an increase in right-turn dominated SPMs (Fig. 4C, red), while left-turn dominated SPMs were barely affected (Fig. 4C, blue).

The change in the recruitment of the SPMs was spatially specific. Thrustdominated SPMs initially occurred throughout the arena (Fig. 4D) but were confined to area I (high thrust speed; Fig. $4 E$ ) and area III after learning (slowzigzagging movement SPM 7; Fig. $4 E$ ). Of particular interest are the modifications in areas II and III. The most prominent change is the emergence of right turndominated SPMs with learning. These SPMs mainly occurred in area II (Fig. 4, compare $F, G$ ) and were typically followed by a slow-thrust SPM (SPM 5) or the slow-zigzagging movement SPM (SPM 7; Fig. $4 E$ ) toward the object. Note that SPM 5 was the most frequent SPM in area III after learning, but did not change as much between learning stages (Fig. 4-1B, available at https://doi.org/ 10.1523/JNEUROSCI.1024-19.2019.f4$1)$. The conditional probability that rightturn SPMs in area II were followed by SPM 7 was $33 \%$, and by SPM 5 was $59 \%$ $\left(\chi^{2}\right.$ test with Monte Carlo permutations, $p<0.0002)$. The zigzagging pattern of
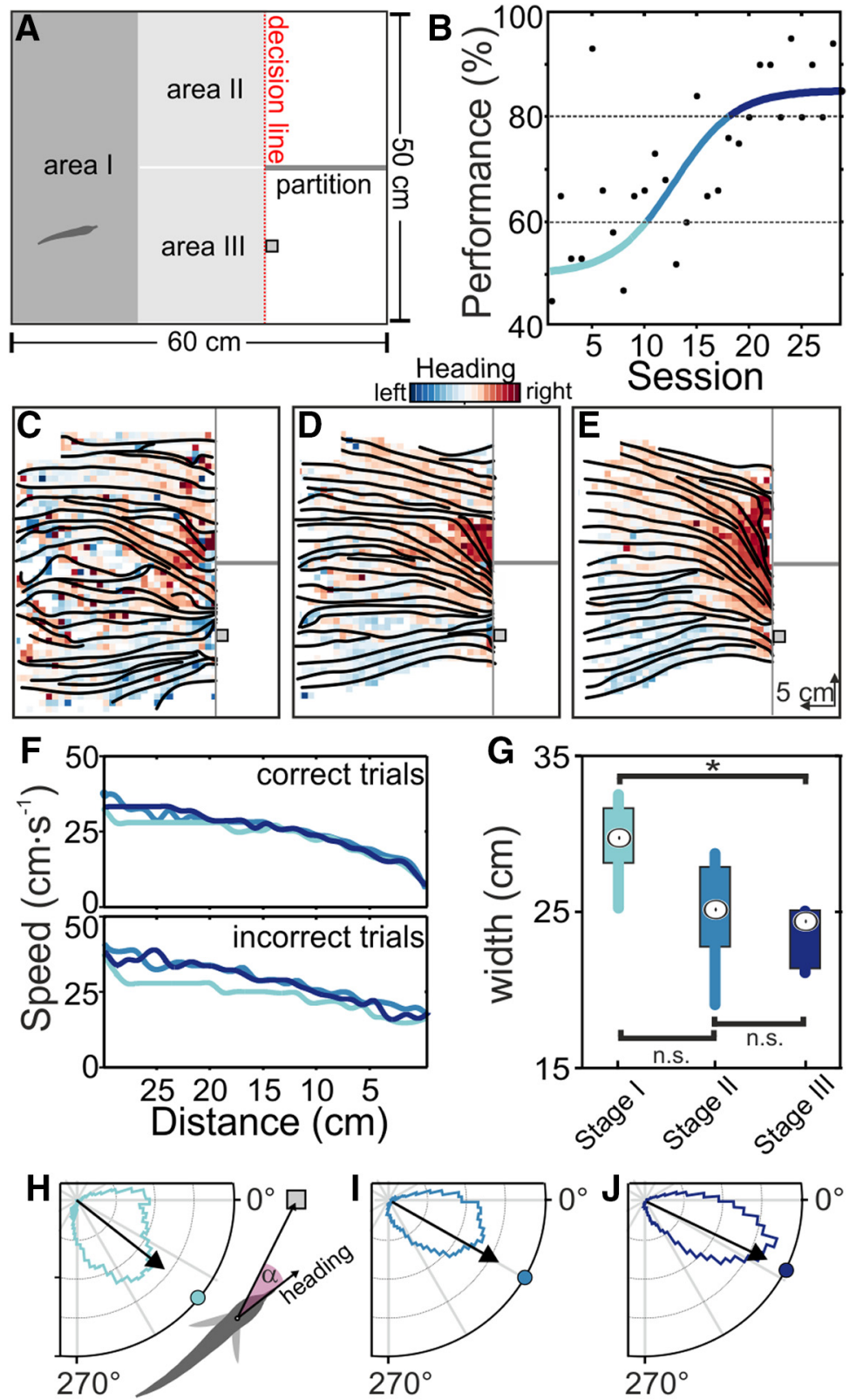

Figure 3. Performance and motor behavior change with learning. $\boldsymbol{A}$, Top view on the experimental arena. Upon lowering the gate that separated the training and the living area, fish entered the arena. Note that the living area is not shown in this figure. The task was to swim into the compartment marked with the metal cube (gray square). Here and in all following figures, the data are presented with the object (square) on the right side of the arena as seen from the view of the fish entering the arena. The arena was partitioned in three areas (I-III) for the later data analysis. B, Psychometric function fitted to the data of one exemplary fish. Here and in the following, the three performance levels used in the analysis are indicated by color (learning stage l: $<60 \%$ of correct decision, light cyan; learning stage II: $>60-80 \%$, dark cyan; learning stage III: $>80 \%$, violet). $\boldsymbol{C}-\boldsymbol{E}$, Top view on the average movements (black lines) during the three stages for the same fish as shown in $\boldsymbol{B}$. The color code indicates the mean heading direction (see sketch in $\boldsymbol{H}$ ). $\boldsymbol{F}$, Median swim speed ( $N=5$ fish) with respect to the distance to the object in correct trials (top; $n=1119$ ) and incorrect trials (bottom; $n=514$ ). $\mathbf{G}$, Boxplots showing the width of the distribution of the fish along the width of the arena (in total $50 \mathrm{~cm}$ ) for all trials $(n=1633$ trials, $N=5$ ). Width was quantified as the range including the 90 th and 10 th percentiles of the data of each individual fish. This measure shows that with learning fish transitioned from exploring the whole width of the arena to a more refined use of the arena that brought them more toward the middle of the arena (Kruskal-Wallis test with Bonferroni post hoc test; stage I vs III, $p=0.03$ ). $\boldsymbol{H}-\boldsymbol{J}$, With learning, fish aligned better with the object (measured from the start of the trajectory until the decision line), as shown here by the mean alignment vector (black arrows, see $\alpha$ in sketch). Colored lines are circular histograms of the raw data. With learning, the mean alignment became more oriented toward the object (Figure 3-1, available at https://doi.org/10.1523/JNEUROSCI.1024-19.2019.f3-1). 

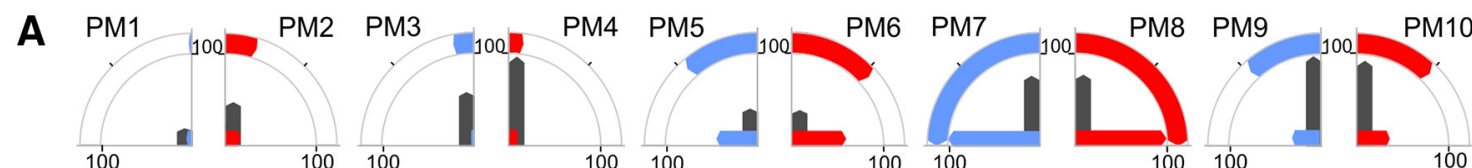

B
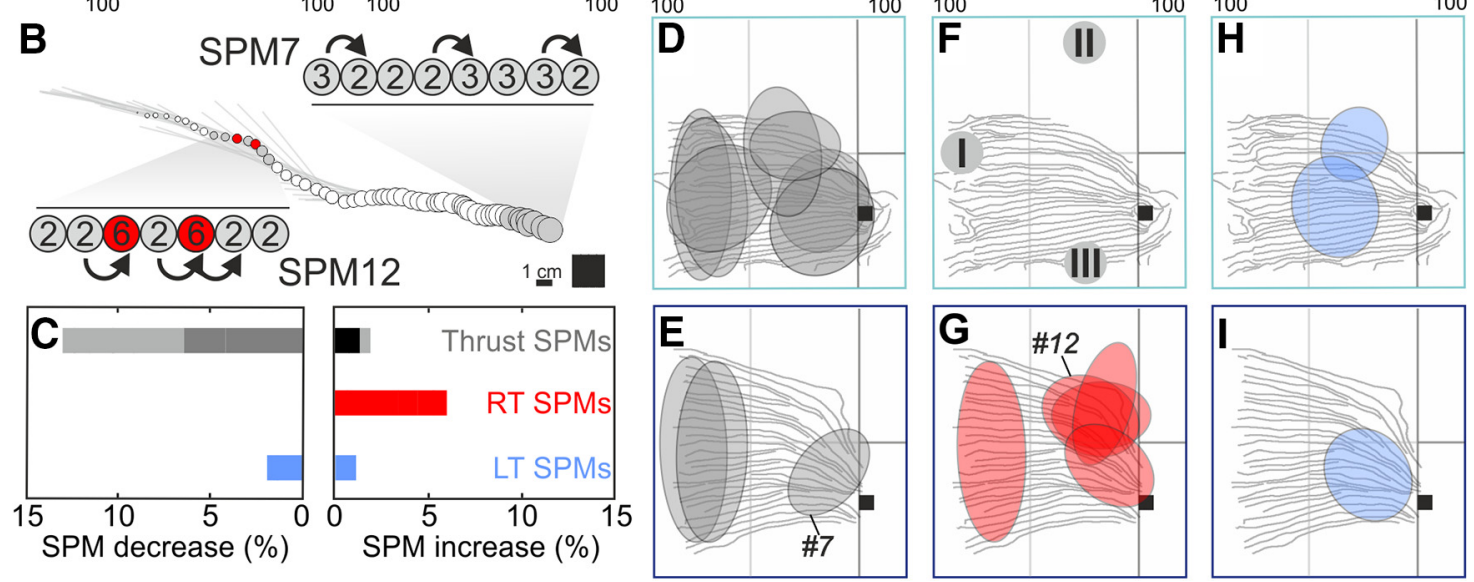

Figure 4. The spatial recruitment of motor patterns (i.e., SPMs) shows pronounced changes in the different learning stages. $A$, Kinematics of the 10 PMs depicting the relative centroid values of thrust slip and yaw. Blue bars indicate slip and yaw components directed to the left, while red bars show the corresponding values for movements to the right. PMs $1-4$ are thrust dominated, while PMs 5-10 are turn dominated. $\boldsymbol{B}$, Exemplary trajectory showing the head position of the fish by the circles and the orientation of its body by the gray lines, while time since the start of the trial is indicated by the size of the circles. Using the transition probabilities between PMs, chains of consecutive motor behaviors are extracted (SPMs). As an example, the kinematic sequence of SPMs 7 and 12 is shown by the series circles where the numbered circles stand for the corresponding PM. C, Relative change of occurrence for the SPMs that showed the strongest change comparing stage I to III (left) and stage III to I (right) for correct trials. Note that low- to medium-thrust SPMs (light and darker gray) decreased, while high-thrust SPMs (black) increased. Right turn (RT)-dominated SPMs (red) were more frequent in stage III. D-I, Spatial distribution of the SPMs shown in ( for learning stages I (top row, D, F and H) and III (bottom row, E, G and I). The colored ellipses are based on 2D Gaussian fits to the spatial distribution of SPM with colors separating thrust-dominated (gray), RT-dominated (red), and left turn (LT)-dominated (blue) SPMs. The numbers in $\boldsymbol{E}$ and $\boldsymbol{G}$ refer to the two SPMs detailed in $\boldsymbol{B}$ (Figure 4-1, available at https://doi.org/10.1523/JNEUROSCI.1024-19.2019.f4-1).
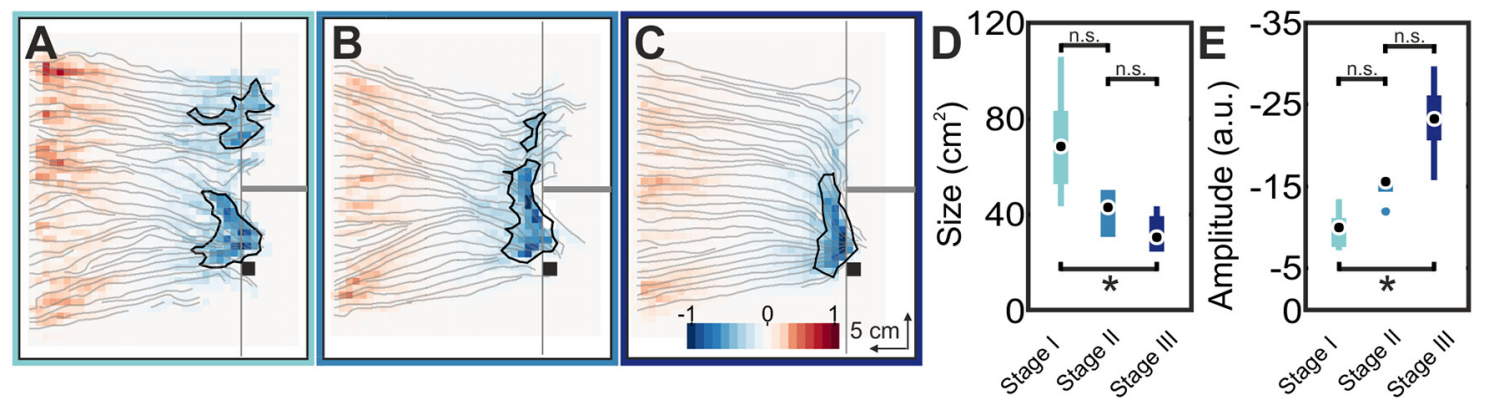

Figure 5. Development of attracting set states with learning. $\boldsymbol{A}-\boldsymbol{C}$, Attractor landscape diagram for the trajectories of an exemplary fish in stages I ( $\boldsymbol{A})$, II (B), and III (C). The color code depicts the attractor value, light solid lines show average trajectories, and black solid lines show the area of the attracting set. Initially, two attracting sets of similar size were present, while attracting sets became more distinct around the object. $D$, Size of the attracting set (see Materials and Methods) shown as boxplots for all fish and learning stages. Attracting set size significantly decreased from stage I to stage III (Mann-Whitney pairwise test with Bonferroni post hoc test: stages I-II, $p=0.13$; stages I-III, $p=0.04$; and stages II-III, $p=0.49$ ). $E$, Same as in $\boldsymbol{D}$ for the attracting set peak amplitude. Peak amplitude increased significantly from stage I to stage III (Mann-Whitney pairwise test with Bonferroni posthoc test: stages I-II, $p=0.13 ;$ stages I-II, $p=0.06 ;$ stages I-III, $p=$ 0.03 ; stages II-III, $p=0.1)$. In all panels, asterisks indicate statistical significance $(p<0.05$; Figure 5-1, available at https://doi.org/10.1523/JNEUROSCI.1024-19.2019.f5-1).

SPM 7 consisted of a succession of left and right PMs, resulting in an average change of the heading of the animals of $3.77 \pm 2.14^{\circ}$ per cycle at a frequency of $\sim 21 \mathrm{~Hz}$ (Fig. $4 B$, SPM 7). Similar to PMs, left turn-dominated SPMs changed less with learning and were found close to the object both before and after learning (Fig. 4 , compare $H, I$ ).

\section{Altered behavior is reflected in the formation of an attracting set}

Altered trajectories may represent overt actions reflecting the internal motor decision-making process necessary to improve the performance with learning. In this context, trajectories leading to behavioral choices have been used to map how motor behavior may represent the formation of internal states like goals (O'Hora et al., 2013; Zgonnikov et al., 2017). Applying this approach to our data, we find that at the start of learning, from any start point the trajectory of the fish will end up within one of the two compartments. These stable endpoints form two attracting sets ("sinks") around the two possible locations of the object (Fig. 5A and, for all fish, see Fig. 5-1, available at https://doi.org/10.1523/ JNEUROSCI.1024-19.2019.f5-1). With learning, a single attracting set emerged at the entry of the reinforced $(S+)$ compartment (Fig. $5 B, C$ ). This attracting set became more distinct with learning (i.e., its size decreased; Mann-Whitney pairwise test with Bonferroni post hoc test: stages I-II, $p=$ 0.13 ; stages I-III, $p=0.04$; and stages II-III, $p=0.49$; Fig. $5 D)$, and its peak amplitude increased (Mann-Whitney pairwise test with Bonferroni post hoc test: stages I-II, $p=0.06$; stages I-III, $p=0.03$; and stages II-III, $p=0.11$; absolute values; Fig. $5 E)$. Overall, these results show that the fish learned to change 

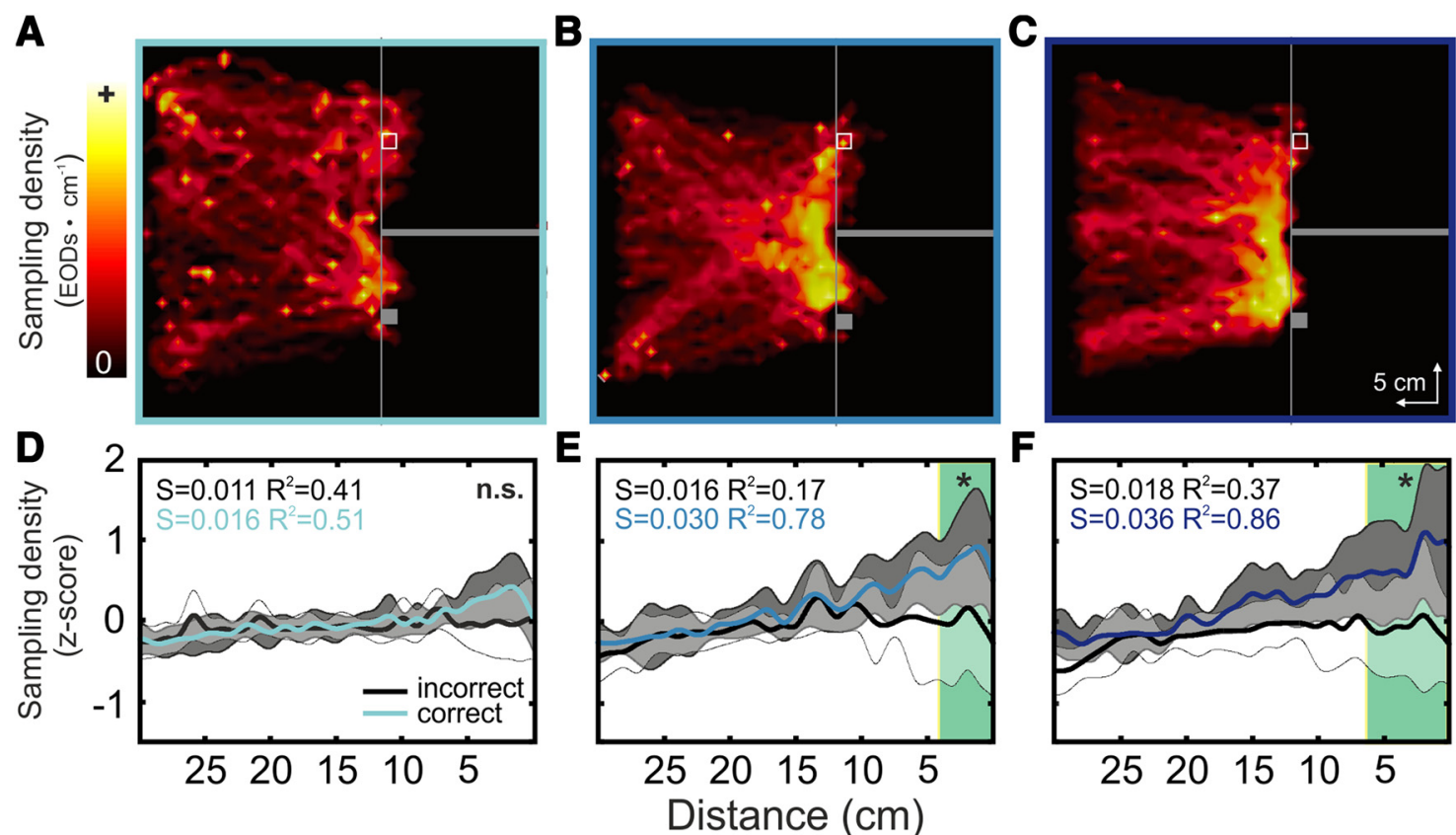

F

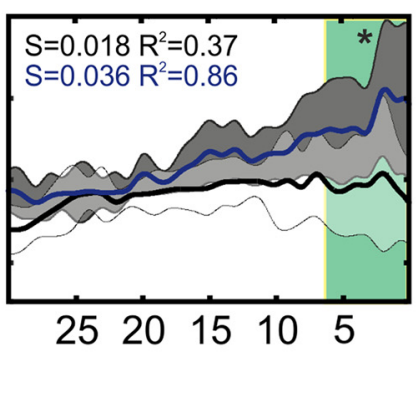

Figure 6. Change of the sampling density with learning. $A-C$, Distribution of the normalized sampling density for the three stages of learning (data from exemplary fish). As in Figure $3 A$, the open square shows the virtual position of the cube, while the solid cube shows the actual position of cubes. $D-F$, Sampling density ( $z$-scored) as a function of distance to the object (correct trials, $n=1119$; colored lines and shaded area depict median and median absolute deviation) and the virtual object (incorrect trials, $n=514$; black lines and light gray outlines show median and median absolute deviation). The slopes and coefficients of determination of the linear fits to the sampling density are shown in colored font for the correct trials and in black font for the incorrect trials. The distances over which sampling density was significantly increased in correct vs incorrect trials are indicated by green-shaded areas (Wilcoxon signed-rank test, $p=2 \mathrm{e}^{-4}-0.0495$ and $7 \mathrm{e}^{-7}-0.02 \mathrm{for}$ stages II and III, respectively; Figure 6-1, available at https://doi.org/10.1523/JNEUROSCI.1024-19.2019.f6-1, and Figure 6-2, available at https://doi.org/10.1523/JNEUROSCI.1024-19.2019.f6-2).

the focus of their behavioral attention from an initially bimodal to a unimodal attracting set.

\section{Sampling behavior adapts alongside kinematics}

The emergence of a single attracting set indicates that the animals learned to attend to and use the object in their motor planning. To understand whether and how this affected the sensory sampling behavior, we calculated the sampling density (number of EODs emitted per distance traveled, EOD $\left.\cdot \mathrm{cm}^{-1}\right)$. Sampling density increased toward the object (Fig. $6 A-C$ and, for all fish, see Fig. 6-1, available at https://doi.org/10.1523/JNEUROSCI.102419.2019.f6-1). This rise was shallow and undistinguishable between correct and incorrect trials in stage I $(t$ test for slopes to linear regression, $p=$ 0.051 ; Fig. $6 D$, compare cyan line, black line). With learning however, the sampling density increase was steeper in correct trials $(t$ test for slopes, $p<0.001$; stages II and III), resulting in higher sampling density values close to the object (Fig. $6 E$, compare dark cyan, black; Wilcoxon signed-rank test for stage II, $p \leq 0.0495$; Fig. $6 F$, compare violet, black; stage III, $p \leq 0.02$ ). The average EOD rate also increased with learning (mean \pm SD: $32.56 \pm 17.14,34.43 \pm 16.18,39.45 \pm 14.61 \mathrm{EODs} \cdot \mathrm{s}^{-1}$, Kruskal-Wallis test with Bonferroni post hoc test: stage I vs II, $p=0.001$; stage I vs III, $p=6.49 \mathrm{e}-31$; stage II vs III, $p=2.85 \mathrm{e}-16$ ). However, EOD rates increased only slightly when the fish approached the decision line (see $z$-scored data in Fig. 6-2, available at https://doi.org/10.1523/ JNEUROSCI.1024-19.2019.f6-2). The increase of the sampling density with proximity to the object thus mainly was due to the reduced swim velocity (compare Figs. 3F, 4, SPM data). This suggests that the region of the attracting set also recruited the highest sampling behavior.

\section{Sensory consequences of behavioral adaptations}

To understand how the spatial changes in sampling density translate to sensory information, we used a biophysical model to calculate the EIs (the afferent sensory input; Pedraja et al., 2016;
Hofmann et al., 2017). From these, we calculated the FI between successive EIs (see Materials and Methods; Fig. 1). FI can be regarded as an upper bound on the variance of the estimation of the cube detectability that a given EI provides. As expected, FI increased with proximity to the cube (Fig. $7 A-C$, top graphs). Interestingly, the increase was significantly stronger in later learning stages (median FI: stage I, $1.90 \cdot 10^{4}$; stage II, $1.64 \cdot 10^{5}$; stage III, $3.32 \cdot 10^{5}$; Kruskal-Wallis test with Bonferroni post hoc test for all comparisons, $p<0.001)$. The distance over which FI was increased between learning stages progressively increased with learning (Fig. 7A-C, bottom, white arc indicates significant areas; stage I vs II, $5 \mathrm{~cm}$; stage I vs III, $6 \mathrm{~cm}$; Kruskal-Wallis test with Bonferroni post hoc test, $p$ values between $1 \cdot 10^{-4}$ and $0.049)$. This shows that the sensory information is enhanced by the sensorimotor adjustments with learning.

\section{Behavioral and physiological detection range}

The mean physiological detection limit for ELL units reported here $(9.2 \mathrm{~mm})$ is similar to those reported for electroreceptor afferents (Szabo and Hagiwara, 1967; Gómez et al., 2004). To obtain an estimate of the detection range on the organismal level (perception) and in context of unrestrained behavior, we tested detection ranges in our experimental tank with fish that had completed training (Fig. $7 D, E$, stage III; see Materials and Methods). This detection threshold (Fig. $7 E$; mean $\pm \mathrm{SD}, 65 \pm 6 \mathrm{~mm} ; N=5$ ) was approximately sevenfold higher than the thresholds determined in physiology. Nonetheless, this behavioral threshold is in agreement with detection range estimates reported using different approaches (onequarter to one-half body length, equivalent to $5-8 \mathrm{~cm}$ in our fish (Push and Moller, 1979; Toerring and Belbenoit, 1979).

We assumed an FI level at the detection limit (Fig. 7F; i.e., 65 mm distance) to be the minimum information that trained fish required to detect the object. Naïve fish (Fig. $7 F$, stage I), in 

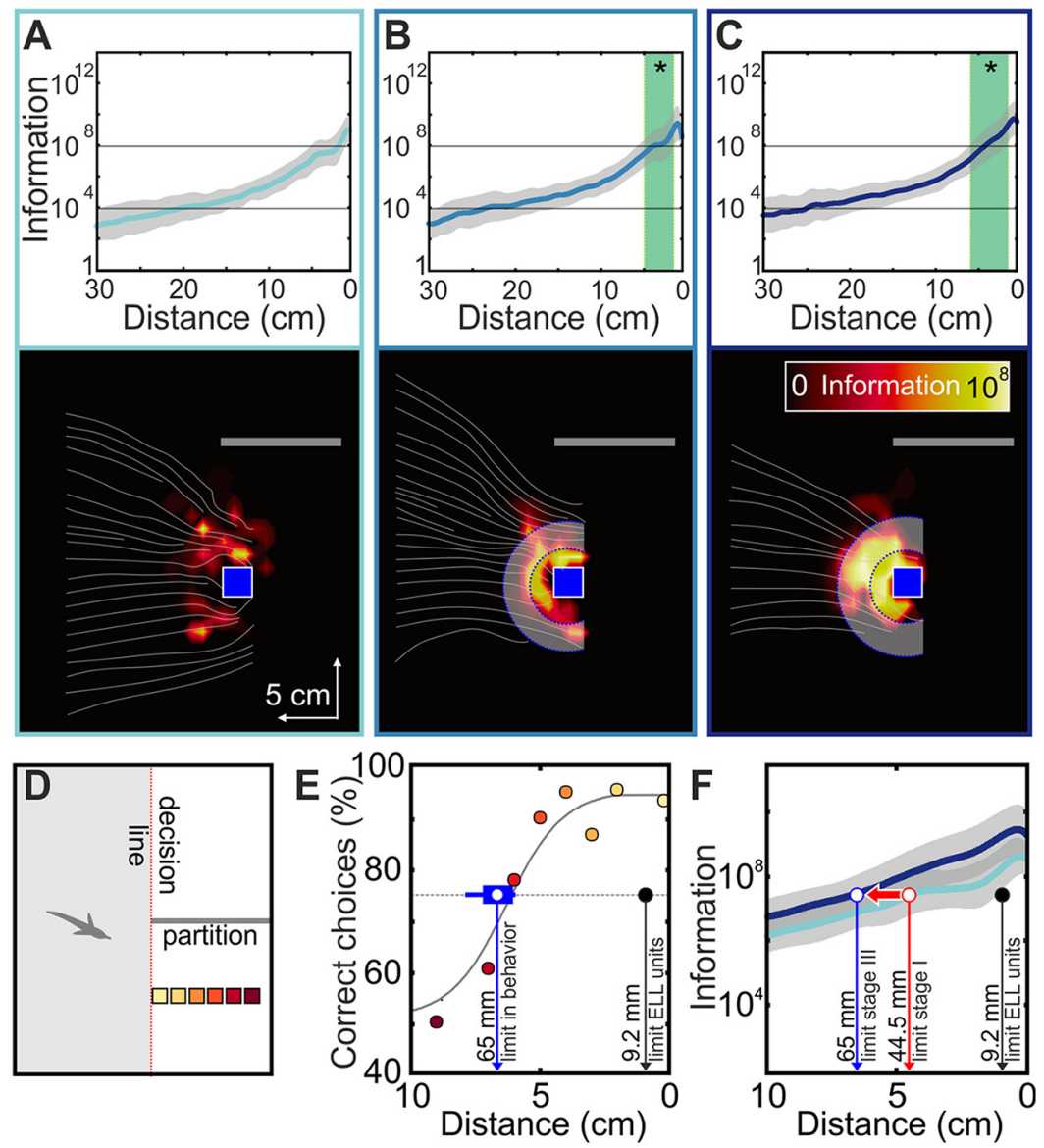

Figure 7. Fisher information increases with object proximity and learning. $\boldsymbol{A}-\boldsymbol{C}$, Top, Fisher information as a function of distance to the cube for the three learning stages (left to right). Colored lines show median, and shaded areas show median absolute deviation. Green boxes show the range over which Fl was significantly increased in successive learning stages (KruskalWallis test with Bonferroni post hoc test, $p<0.05$ ). Note the log-scale of the $y$-axis. Bottom, Spatial representation of the same data. Color code depicts the values of Fl, and light gray lines show the average trajectories superimposed. White semicircles show the range for the difference in Fl showed in top panels. These data confirm that the behavioral adaptions observed during learning in fact impact the available information to the fish. $\boldsymbol{D}$, Top view of the experimental arena showing how the detection limit was measured in behavioral experiments for trained fish (i.e., after stage III). For this, the cube was presented with increasing offset from its original position at the decision line (yellow to red). $\boldsymbol{E}$, Psychometric performance (correct choices as a function of distance between cube and decision line) of one fish. The detection limit was determined as the distance of the object from the decision line where the performance (sigmoidal fit) fell to $<75 \%$. The detection limits for all fish are summarized by the data depicted by the blue boxplot (mean, $65 \mathrm{~mm}$ ). $\boldsymbol{F}$, Fisher information as a function of the distance of the fish to the cube in stages I (cyan) and III (blue). The Fl value at the average behavioral detection limit $(65 \mathrm{~mm}$; see $\boldsymbol{E}$ ) is indicated by the white circle with blue edge. To reach a similar Fl level during stage I, fish needed get closer to the object ( $44.5 \mathrm{~mm}$; white circle with red edge). The red horizontal arrow indicates this 1.4-fold increase in sensory range following sensorimotor learning. The detection limit inferred from the electrophysiological results was much lower $(9.2 \mathrm{~mm} ; \boldsymbol{E}, \boldsymbol{F}$, black circles).

comparison, needed to get significantly closer to the object $(44.5$ $\mathrm{mm}$ ) to achieve a similar FI level. This still is $\sim 4.8$ times better than the physiological threshold. We interpret this to reflect the contribution of different neuronal mechanisms in coping with challenging signal-to-noise conditions (e.g., population coding, feedback influence; see Discussion), and it cannot be revealed in the single-cell recordings conducted here. The additional 1.4-fold increase of the threshold with learning likely is due to the electromotor adjustments reported here. Our study thus provides a first estimate of the efficiency of the neuronal mechanisms in dealing with the low signal-to-noise problem and, in addition, supports the hypothesis that an active choice of sensing behavior can substantially contribute to improve the detectability of weak stimuli.

\section{Linking motor patterns with information and electromotor responses}

To further understand the means by which behavioral adjustments can increase sensory information, we turned to a simple theoretical abstraction. Figure 9 shows the amplitude of the electric images of a fish approaching a metal cube for different scenarios at a constant sampling density: once for the fish approaching the cube frontally with the EI being focused on the head (Fig. 8A), once for an angled approach to the edge of the cube that places the EI more laterally (Fig. $8 B$ ), and once for a straight approach to the side of the cube that again places the EI laterally (Fig. 8C). The increase of electric image amplitude is higher in the later cases (Fig. 8 , black lines). This translates into higher FI (Fig. 8, blue lines) between successive sampling events. The EI gradient is less steep when an object is frontally approached (Fig. 8; Hofmann et al., 2017, their Fig. 4). Thus, by moving the electric image over the head region, fish could potentially exploit the heterogeneity of the electric field geometry and prereceptor mechanisms to increase the gradient and thereby the sensory information. To explore this, we modeled EIs and calculated FI for a virtual scanning behavior in which the EI is moved repetitively from the frontal to the lateral side of the body side (Fig. $8 D$ ). This results in a strongly increased FI. This virtual approach is reminiscent of SPM 7, a kinematic pattern selectively recruited after learning. This suggests that the variation of the position of the electric image due to rotatory movements in the approach may be crucial in enhancing the information the animal can obtain with this behavior.

To analyze whether electromotor (i.e., sensory) behavior was associated with specific kinematic patterns or motion primitives (i.e., PM or SPMs), we analyzed the likelihood that SPMs were associated with a specific electromotor display. E-scans are transient rises of the EOD frequency (Post and von der Emde, 1999; Jun et al., 2016) that occur when the sensory input deviates from the recent sensory past (Caputi et al., 2003). E-scans were found in $5.8 \pm 4.5 \%$ (mean \pm SD) of the SPMs. The three SPMs that were specifically elevated after learning in areas II and III also had significantly elevated E-scan probabilities (SPM 7, 25\%; SPM 12, 20\%; SPM 13, 14\%; $\chi^{2}$ test with Monte Carlo permutations, $p<0.01$; Fig. $9 A, B)$. Likewise, the three SPMs specific to area I also had elevated E-scan levels (SPM 1, 24\%; SPM 9, $16 \%$; SPM 10, 30\%; $\chi^{2}$ test with Monte Carlo permutations, $p<0.01$; Fig. $9 A, B)$.

To analyze whether E-scans occurred linked to specific kinematics and to reveal their effect on the sensory information, we calculated PM-triggered averages (see Materials and Methods). 
No change was found in E-scan frequency following right-turn PMs in straight approaches passing from area I to III directly (Fig. 9C, bottom). However, E-scan probabilities increased for right-turn PMs when fish transverse from area I to II to III (diagonal approach; Fig. 9D, bottom; Kruskal-Wallis test with Bonferroni post hoc test, $p=0.02)$. In both cases, Fisher information was elevated after the turns (Fig. 9C,D, top; Kruskal-Wallis test with Bonferroni post hoc test: direct, $p=0.02$; diagonal, $p=0.005)$. No such relation for E-scans or Fisher information was found for left-turn PMs (for direct and diagonal approaches, see Fig. 9-1 $A, B$, available at https://doi.org/10.1523/JNEUROSCI. 1024-19.2019.f9-1) or high-thrust PMs (for direct and diagonal approaches, see Fig. 9-1C,D, available at https://doi.org/ 10.1523/JNEUROSCI.1024-19.2019.f91), while low-thrust PMs again led to an increased FI (Fig. 9-1E,F, available at https://doi.org/10.1523/JNEUROSCI. 1024-19.2019.f9-1; Kruskal-Wallis test with Bonferroni post hoc test: direct, $p=$ 0.002; diagonal, $p=0.008)$. This indicates that the sequence of right turn-dominated SPMs and the consecutive slow-zigzagging or gliding SPMs in area III (Figs. 4, 8) are essential stereotyped motor patterns that are linked with a stereotyped electromotor response that together result in an improved sensory information (Fig. 5).

In summary, we have established that the object detection range for ELL neurons under static conditions is significantly lower than the behavioral detection range. While learning the task, fish alter the electromotor behavior in a coordinate manner that results in enhanced information levels. Electromotor learning, in addition to neuronal mechanisms, thus can be an important factor in enhancing the sensory range.

\section{Discussion}

To which degree sensing depends on the mutual interactions of sensory and motor processes is a fundamental question in neuroscience (Gordon et al., 2011). Examples for the contribution of motor behavior include situations where movements stabilize sensory input, or directly enhance or generate sensory information. In either case, movement is an integral part of sensing. This is particularly evident in active sensory systems, where motor control and sensing are directly connected (Ahissar and Assa, 2016).

The experimentally obtained detection range of ELL units reported here likely underestimates the natural sensitivity of ELL units. This is because, among other factors, the ELL sensitivity likely depends on feedback (Chacron et al., 2005; Sawtell and Bell, 2008; Clarke and Maler, 2017; Enikolopov et al., 2018; Hofmann and Chacron, 2019) as well as feedforward mechanisms and natural behavior. Furthermore, population activity is likely a better determinant of perception and behavior than the activity of single neurons (Pitkow and Angelaki, 2017; Runyan et al., 2017; Ni et al., 2018). It is also possible that stimulus detection is encoded in a parallel pathway (McGillivray et al., 2012; Huang and Chacron, 2016), where the selective decoding of information of ELL efferents with high detection ranges could improve the percep- tual range. The reported detection limits thus provide a lower estimate of the performance under natural conditions. Future studies should validate these results both for single units and on the population level in freely behaving fish.

In our experiments, fish learned to find and swim to the area marked by a metal cube. Apart from slight variations in the time fish took to acquire this task, all animals learned to solve it and further did so using very similar sensorimotor patterns. This involved a refined use of the arena, emergence of targeted turn patterns, and an increase of the sampling density in the vicinity of the object. This is comparable to the kinematic data published for this species (Hofmann et al., 2017) for spontaneous approaches toward novel objects where alterations of the sensorimotor behavior were found to contribute to the shaping of the sensory input, leading to the emergence of depth information. Notably, in the present study, changes of the motor patterns occurred both within and outside of the detection range of the electric system, suggesting that they are driven by sensory information (i.e., reactive control) as well as internalized predictions. Together, this resulted in changes of the spatial attention of the animals with learning. A particular case concerns the different slopes of the sampling density in correct and incorrect trials. As these happen before sensory information becomes available, it may indicate a switch between two sampling modes. Hypothetically, this could be explained as an optimized foraging behavior where fish actively seek the rewarded compartment most of the time but occasionally put less effort into localizing the object, probably to seek out the area of low reward/food probability.

Wave-type weakly electric fish position themselves with a preferred distance to transversely moving objects, where the slope of the signal is highest, corresponding to the Fisher optimal distance (Clarke et al., 2015). Similarly, echolocating bats track targets by aiming their acoustic beam to hit the object off center (i.e., where the slope in the acoustic beam amplitude is the highest; Yovel et al., 2010). In addition, unconditioned approaches to objects of $G$. 

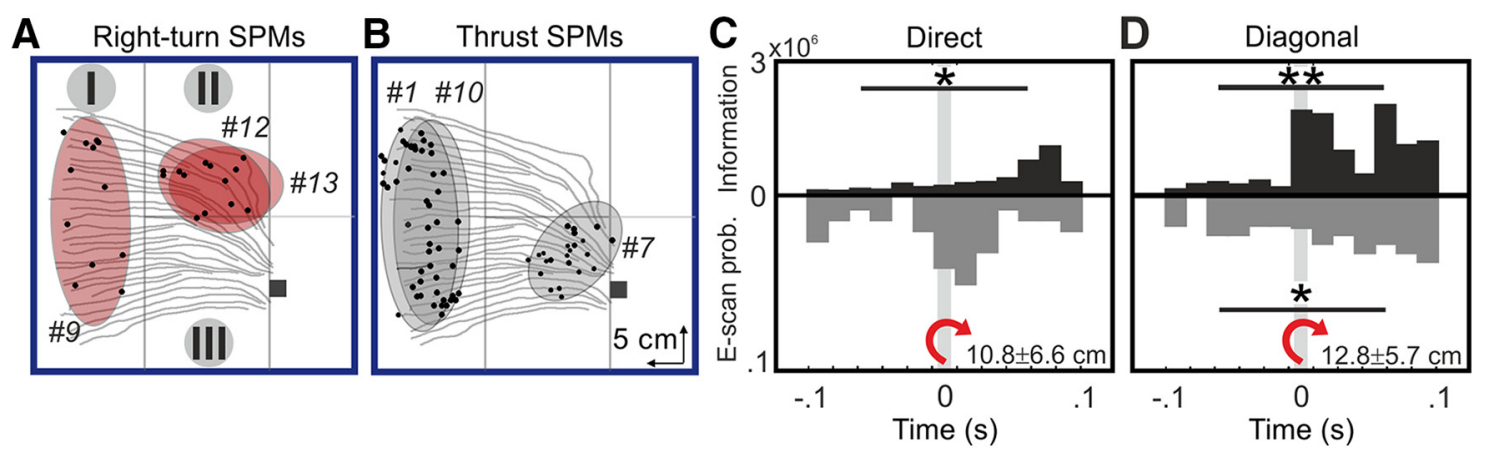

Figure 9. Object-directed turns increased sensory information and resulted in transient increases of sampling rates. $A, B$, Spatial distributions of the SPMs (see Materials and Methods) with the strongest change between learning stages I and III, which also showed an elevated E-scan probability (range, 14-30\%; 25\% for SPM 7). $\boldsymbol{A}$, Right turn-dominated SPMs (red). B, Thrust-dominated SPMs (gray). In both plots, dots indicate the positions of E-scans. Light gray lines are average trajectories. C, Fisher information (top) and E-scan probability (bottom) triggered on right turns (PM based, PM occurrence at time 0 ) during direct approaches (see $C$. FI was significantly increased after the right turn (positive time values, Kruskal-Wallis test with Bonferroni post hoc test: direct, $p=$ 0.02). D, Same as C but for diagonal trajectories. Fl and E-scan probability were significantly increased after the right turn (Kruskal-Wallis test with Bonferroni post hoc test: $\mathrm{Fl}, p=0.005 ; \mathrm{E}-\mathrm{scan}$ probability, $p=0.02$ ). In both panels, light gray numbers indicate the distance at which turns were observed on average (mean \pm SD; Figure 9-1, available at https://doi.org/10.1523/ JNEUROSCI.1024-19.2019.f9-1).

petersii were shown to be oriented along the field gradient in a manner that may enable fish to determine the distance to their object (Hofmann et al., 2017). These examples show that in active-sensing animals, the most informative aspects of the sensory input often are linked to the highest gradient in the carrier signal.

We found that, with learning, fish altered their sensorimotor behavior in a manner that increased sensory information, likely resulting in the improved detection range after learning. A potential strategy to achieve this is bringing the electric image onto the part of the sensory surface where it causes the strongest change in EI amplitude, thus increasing the gradient in the temporal afferent signal (Fig. 8A-C; Babineau et al., 2006). We here suggest a further option in which fish actively move the sensory input over their foveal head region, a strategy that will significantly increase information about a target (Fig. 8D). Fish did focus the input to the head, but not to the front. They further recruited a motor prototype consisting of a slow zigzagging toward the object. This indicates that this third option may indeed be used to enhance sensory input. Future studies, using refined videography, should thus address whether the positioning of the electric images on the foveal head region is different in tasks that demand the fish to detect an object versus one where object features need to be discriminated. Likewise, electrophysiological studies on freely behaving fish are required to directly address the predicted improvement of the neuronal detection limits through specific learned motor behaviors.

The ability to actively regulate the sensory input is emerging as a general research question. A particularly striking example for this comes from a wave-type weakly electric Gymnotiform fish that engages in energetically inefficient foraging to enlarge the electrosensory range (Snyder et al., 2007; MacIver et al., 2010; Biswas et al., 2018). An alternative strategy, an increase of the signal amplitude, seems to be common in echolocating toothed whales and bats (Madsen and Surlykke, 2013). Contrary to these species, weakly electric fish, likely due to the energetic costs of maintaining the EOD (Markham et al., 2016) and the spherical dissipation of the energy (Nelson and Maciver, 1999), appear to have favored motor adjustments as a means to increase the sensory range. These motor adjustments include a reduced swim speed close to informative objects in the environment that result in elevated sampling density rates (see Results; Jun et al., 2016). In addition to a reduction in energy expenditure, this motor- dependent modulation of the sampling density rate might also enable an easier change in swim direction upon encountering informative regions in space (e.g., B-scans; Jun et al., 2016). Where studied, rises of the sampling density were coupled to reduced swim speeds. Elevated sampling density rates thus might also reflect motor necessities inherent to the motor behavior. While our study cannot conclusively disambiguate motor and sensory objectives, a recent study by Fotowat et al. (2019) provides strong support to the view that sampling density is crucially involved in the active regulation of information transmission and sensing. Recordings from neurons in the dorsal pallium of freely swimming Gymnotiform fish revealed neurons that were specifically active directly after elevated sampling density rates. This is consistent with the interpretation that changes of motor and electromotor activity (here, the sampling density rate) can result in an improved neuronal representation. Future studies are needed to further separate the sensory and motor objectives of changes in the sampling density.

A second behavioral parameter that was adjusted as fish learned the task was turning (during approaches that initially targeted the wrong compartment). These turns (SPMs 12, 13, and 14) orient the animal to the informative region (area III), where another set of SPMs (5 and 7) was recruited. The turns predominantly occurred outside of the neuronal detection range of the object and were followed by elevated E-scans rates. This argues for a coordinate control of motor and electromotor behaviors that together result in an increase of the information near an object.

Similar to head scans of rodents (Monaco et al., 2014), transient rises of the sampling frequency have been considered to serve special functions in the formation of spatial memories (Jun et al., 2016) and have been implicated further as overt displays of sensory expectations (Moller, 1995). Indeed, startle responses of the EOD were among the first evidence for the hypothesis (Heiligenberg, 1980, 1988) that weakly electric fish can compare current afferent input against an internal reference or memory of the past afference (Hall et al., 1995). E-scans linked to these turns might be overt displays that signal a match between expected and perceived sensory input (Moller, 1995). Finally, using zigzagging in the final approach may provide further additional sensory information. An alternative explanation of E-scans being associated with turns is that the animals seek to maintain the sensory change between successive sampling events within a preferred 
range. The transient increase of the EOD rate may thus serve to compensate the steep change of the sensory input that follows the turns. Further studies that allow clamping the level of sensory change in freely behaving fish are required to investigate this hypothesis. Evidence for an active buffering of the level of sensory input has recently been shown for a different sensorimotor behavior in a wave-type weakly electric fish (Biswas et al., 2018). The finding that motor behaviors, which are relevant in regulating the sensory flow, are fairly stereotyped will also enable direct studies of their role in neuronal processing, as these motor behaviors can be easily quantified in the ongoing behavior and thus allow direct connection with neuronal data (Kern et al., 2001).

Active sensing in its most common form involves the generation of movements. Whether and how these are controlled with respect to their sensory corollaries is mostly unknown. Our results show that motor control can be an active component of sensory learning. Similar effects are to be expected in other sensory systems, particularly near-range and active sensory systems. A better understanding of the strategies guiding sensory learning thus will likely lead to a better understanding of sensorimotor integration, variation of behavior in natural contexts, and learning in general. Active shaping of the sensory flow in general may be of further interest in technical systems, where acoustic beam forming of field shaping can be used to acquire different information using different sensorimotor configurations when required.

\section{References}

Ahissar E, Assa E (2016) Perception as a closed-loop convergence process. Elife 5:e12830.

Babineau D, Longtin A, Lewis JE (2006) Modeling the electric field of weakly electric fish. J Exp Biol 209:3636-3651.

Bell CC, Zakon H, Finger TE (1989) Mormyromast electroreceptor organs and their afferent fibers in mormyrid fish: I. Morphology. J Comp Neurol 286:391-407.

Bell CC, Meek J, Yang JY (2005) Immunocytochemical identification of cell types in the mormyrid electrosensory lobe. J Comp Neurol 483:124-142.

Bell CC, Han V, Sawtell NB (2008) Cerebellum-like structures and their implications for cerebellar function. Annu Rev Neurosci 31:1-24.

Biswas D, Arend LA, Stamper SA, Vágvölgyi BP, Fortune ES, Cowan NJ (2018) Closed-loop control of active sensing movements regulates sensory slip. Curr Biol 28:4029-4036.e4.

Brainard MS, Doupe AJ (2013) Translating birdsong: songbirds as a model for basic and applied medical research. Annu Rev Neurosci 36:489-517.

Braun E, Geurten B, Egelhaaf M (2010) Identifying prototypical components in behaviour using clustering algorithms. PLoS One 5:e9361.

Caputi AA, Aguilera PA, Castelló ME (2003) Probability and amplitude of novelty responses as a function of the change in contrast of the reafferent image in G. carapo. J Exp Biol 206:999-1010.

Chacron MJ, Maler L, Bastian J (2005) Feedback and feedforward control of frequency tuning to naturalistic stimuli. J Neurosci 25:5521-5532.

Charlesworth JD, Tumer EC, Warren TL, Brainard MS (2011) Learning the microstructure of successful behavior. Nat Neurosci 14:373-380.

Ciali S, Gordon J, Moller P (1997) Spectral sensitivity of the weakly discharging electric fish gnathonemus petersi using its electric organ discharges as the response measure. J Fish Biol 50:1074-1087.

Clarke SE, Maler L (2017) Feedback synthesizes neural codes for motion. Curr Biol 27:1356-1361.

Clarke SE, Longtin A, Maler L (2015) The neural dynamics of sensory focus. Nat Commun 6:8764.

Enikolopov AG, Abbott LF, Sawtell NB (2018) Internally generated predictions enhance neural and behavioral detection of sensory stimuli in an electric fish. Neuron 99:135-146.e3.

Fotowat H, Lee C, Jun JJ, Maler L (2019) Neural activity in a hippocampuslike region of the teleost pallium is associated with active sensing and navigation. Elife 8:e44119.

Friston K (2010) The free-energy principle: a unified brain theory? Nat Rev Neurosci 11:127-138.
Gellermann LW (1933) Chance orders of alternating stimuli in visual discrimination experiments. Pedagog Semin J Genet Psychol 42:206-208.

Geurten BR, Kern R, Braun E, Egelhaaf M (2010) A syntax of hoverfly flight prototypes. J Exp Biol 213:2461-2475.

Gómez L, Budelli R, Grant K, Caputi AA (2004) Pre-receptor profile of sensory images and primary afferent neuronal representation in the mormyrid electrosensory system. J Exp Biol 207:2443-2453.

Gordon G, Kaplan DM, Lankow B, Little DY, Sherwin J, Suter BA, Thaler L (2011) Toward an integrated approach to perception and action: conference report and future directions. Front Syst Neurosci 5:20.

Gordon G, Fonio E, Ahissar E (2014) Emergent exploration via novelty management. J Neurosci 34:12646-12661.

Hall C, Bell C, Zelick R (1995) Behavioral evidence of a latency code for stimulus intensity in mormyrid electric fish. J Comp Physiol A 177:29-39.

Heiligenberg W (1980) The jamming avoidance response in the weakly electric fishEigenmannia. Naturwissenschaften 67:499-507.

Heiligenberg W (1988) Electrosensory Maps Form a Substrate for the Distributed and Parallel Control of Behavioral Responses in Weakly Electric Fish. Brain Behav Evol 31:6-16.

Hofmann V, Chacron MJ (2019) Novel functions of feedback in electrosensory processing. Front Integr Neurosci 13:52.

Hofmann V, Sanguinetti-Scheck JI, Gómez-Sena L, Engelmann J (2013) From static electric images to electric flow: towards dynamic perceptual cues in active electroreception. J Physiol Paris 107:95-106.

Hofmann V, Geurten BR, Sanguinetti-Scheck JI, Gómez-Sena L, Engelmann J (2014) Motor patterns during active electrosensory acquisition. Front Behav Neurosci 8:186.

Hofmann V, Sanguinetti-Scheck JI, Gómez-Sena L, Engelmann J (2017) Sensory flow as a basis for a novel distance cue in freely behaving electric fish. J Neurosci 37:302-312.

Hollmann V, Engelmann J, Gómez-Sena L (2016) A quest for excitation: theoretical arguments and immunohistochemical evidence of excitatory granular cells in the ELL of Gnathonemus petersii. J Physiol Paris 110: 190-199.

Huang CG, Chacron MJ (2016) Optimized parallel coding of second-order stimulus features by heterogeneous neural populations. J Neurosci 36 : 9859-9872.

Jun JJ, Longtin A, Maler L (2016) Active sensing associated with spatial learning reveals memory-based attention in an electric fish. J Neurophysiol 115:2577-2592.

Kern R, Petereit C, Egelhaaf M (2001) Neural processing of naturalistic optic flow. J Neurosci 21:RC139.

Lissmann HW, Machin KE (1958) The mechanism of object location in Gymnarchus niloticus and similar fish. J Exp Biol 35:457-486.

Little DY, Sommer FT (2013) Learning and exploration in actionperception loops. Front Neural Circuits 7:37.

Loewenstein G (1994) The psychology of curiosity: a review and reinterpretation. Psychol Bull 116:75.

MacIver MA, Patankar NA, Shirgaonkar AA (2010) Energy-information trade-offs between movement and sensing. PLoS Comput Biol 6:e1000769.

Madsen PT, Surlykke A (2013) Functional convergence in bat and toothed whale biosonars. Physiology 28:276-283.

Markham MR, Ban Y, McCauley AG, Maltby R (2016) Energetics of sensing and communication in electric fish: a blessing and a curse in the anthropocene? Integr Comp Biol 56:889-900.

McGillivray P, Vonderschen K, Fortune ES, Chacron MJ (2012) Parallel coding of first- and second-order stimulus attributes by midbrain electrosensory neurons. J Neurosci 32:5510-5524.

Metzen MG, Engelmann J, Bacelo J, Grant K, von der Emde G (2008) Receptive field properties of neurons in the electrosensory lateral line lobe of the weakly electric fish, Gnathonemus petersii. J Comp Physiol A Neuroethol Sens Neural Behav Physiol 194:1063-1075.

Metzen MG, Huang CG, Chacron MJ (2018) Descending pathways generate perception of and neural responses to weak sensory input. PLoS Biol 16:e2005239.

Migliaro A, Caputi AA, Budelli R (2005) Theoretical analysis of prereceptor image conditioning in weakly electric fish. PLoS Comput Biol 1:123-131.

Miller LM, Silverman Y, MacIver MA, Murphey TD (2016) Ergodic exploration of distributed information. IEEE Trans Robot 32:36-52. 
Moller P (1995) Electric fishes: history and behavior. London: Chapman and Hall.

Monaco JD, Rao G, Roth ED, Knierim JJ (2014) Attentive scanning behavior drives one-trial potentiation of hippocampal place fields. Nat Neurosci 17:725-731.

Nelson ME, Maciver MA (1999) Prey capture in the weakly electric fish Apteronotus albifrons: sensory acquisition strategies and electrosensory consequences. J Exp Biol 202:1195-1203.

Ni AM, Ruff DA, Alberts JJ, Symmonds J, Cohen MR (2018) Learning and attention reveal a general relationship between population activity and behavior. Science 359:463-465.

O’Hora D, Dale R, Piiroinen PT, Connolly F (2013) Local dynamics in decision making: the evolution of preference within and across decisions. Sci Rep 3:2210.

Pedraja F, Aguilera P, Caputi AA, Budelli R (2014) Electric imaging through evolution, a modeling study of commonalities and differences. PLoS Comput Biol 10:e1003722.

Pedraja F, Perrone R, Silva A, Budelli R (2016) Passive and active electroreception during agonistic encounters in the weakly electric fish Gymnotus omarorum. Bioinspir Biomim 11:065002.

Pedraja F, Hofmann V, Lucas KM, Young C, Engelmann J, Lewis JE (2018) Motion parallax in electric sensing. Proc Natl Acad Sci U S A 115: 573-577.

Pitkow X, Angelaki DE (2017) inference in the brain: statistics flowing in redundant population codes. Neuron 94:943-953.

Post N, von der Emde G (1999) The "novelty response" in an electric fish response properties and habituation. Physiol Behav 68:115-128.

Poteser M, Kral K (1995) Visual distance discrimination between stationary targets in praying mantis: an index of the use of motion parallax. J Exp Biol 198:2127-2137.

Push S, Moller P (1979) Spatial aspects of electrolocation in the mormyrid fish, Gnathonemus petersii. J Physiol Paris 75:355-357.

Rother Malamud, DD (2003) Simulación de imágenes eléctricas en peces eléctricos de descarga débil. Master thesis (No. 519.87:597 ROT).

Rother D, Migliaro A, Canetti R, Gómez L, Caputi A, Budelli R (2003) Electric images of two low resistance objects in weakly electric fish. Biosystems 71:169-177.
Runyan CA, Piasini E, Panzeri S, Harvey CD (2017) Distinct timescales of population coding across cortex. Nature 548:92-96.

Sanguinetti-Scheck JI, Pedraja EF, Cilleruelo E, Migliaro A, Aguilera P, Caputi AA, Budelli R (2011) Fish geometry and electric organ discharge determine functional organization of the electrosensory epithelium. PLoS One 6:e27470.

Sawtell NB, Bell CC (2008) Adaptive processing in electrosensory systems: links to cerebellar plasticity and learning. J Physiol Paris 102:223-232.

Sawtell NB, Williams A, Bell CC (2005) From sparks to spikes: information processing in the electrosensory systems of fish. Curr Opin Neurobiol 15:437-443.

Sawtell NB, Williams A, Roberts PD, von der Emde G, Bell CC (2006) Effects of sensing behavior on a latency code. J Neurosci 26:8221-8234.

Silverman Y, Miller LM, MacIver MA, Murphey TD (2013) Optimal planning for information acquisition. In: 2013 IEEE/RSJ international conference on intelligent robots and systems, pp 5974-5980. New York: Institute of Electrical and Electronics Engineers.

Snyder JB, Nelson ME, Burdick JW, Maciver MA (2007) Omnidirectional sensory and motor volumes in electric fish. PLoS Biol 5:e301.

Szabo T, Hagiwara S (1967) A latency-change mechanism involved in sensory coding of electric fish (mormyrids). Physiol Behav 2:331-335.

Toerring MJ, Belbenoit P (1979) Motor programmes and electroreception in mormyrid fish. Behav Ecol Sociobiol 4:369-379.

Toerring MJ, Moller P (1984) Locomotor and electric displays associated with electrolocation during exploratory behavior in mormyrid fish. Behav Brain Res 12:291-306.

van Beers RJ, Baraduc P, Wolpert DM (2002) Role of uncertainty in sensorimotor control. Philos Trans R Soc Lond B Biol Sci 357:1137-1145.

Wolpert DM, Landy MS (2012) Motor control is decision-making. Curr Opin Neurobiol 22:996-1003.

Wu HG, Miyamoto YR, Gonzalez Castro LNG, Ölveczky BP, Smith MA (2014) Temporal structure of motor variability is dynamically regulated and predicts motor learning ability. Nat Neurosci 17:312-321.

Yovel Y, Falk B, Moss CF, Ulanovsky N (2010) Optimal localization by pointing off axis. Science 327:701-704.

Zgonnikov A, Aleni A, Piiroinen PT, O’Hora D, di Bernardo M (2017) Decision landscapes: visualizing mouse-tracking data. R Soc Open Sci 4:170482. 